\title{
Effect of exercise on epicardial fat in adults: a protocol for systematic review and meta-analyses
}

Leonardo Roever ${ }^{1 *}$, Elmiro Santos Resende ${ }^{1}$, Angélica Lemos Debs Diniz ${ }^{1}$, Nilson Penha-Silva ${ }^{1}$, João Lucas O’Connell ${ }^{1}$, Fernanda Rodrigues de Souza $^{1}$, Poliana Rodrigues Alves Duarte ${ }^{1}$, Leandro Teixeira Paranhos Lopes ${ }^{1}$, Paulo Fernando Silva Gomes ${ }^{1}$, Hugo Ribeiro Zanetti ${ }^{2}$, Anaisa Silva Roerver-Borges ${ }^{2}$, Fernando César Veloso ${ }^{1}$, Thiago Montes Fidale ${ }^{1}$, Antonio Casella-Filho ${ }^{3}$, Paulo Magno Martins Dourado ${ }^{3}$, Antonio Carlos Palandri Chagass, ${ }^{3,4}$, Sadeq Ali-Hasan-Al-Saegh ${ }^{5}$, Paulo Eduardo Ocke Reis ${ }^{6}$, Rogério de MeloCosta Pinto ${ }^{1}$, Gustavo BF Oliveira ${ }^{7}$, Álvaro Avezum, Mansueto Neto ${ }^{8}$, André Durães ${ }^{8}$, Rose Mary Ferreira Lisboa da Silva', Antonio José Grande ${ }^{10}$, Celise Denardi ${ }^{11}$, Renato Delascio Lopes ${ }^{12}$, Nitesh Nerlekar $^{13}$, Shahab Alizadeh ${ }^{14}$, Adrian V Hernandez ${ }^{15}$, Maria Inês da Rosa ${ }^{16}$, Gary Tse ${ }^{16}$, Tong Liu ${ }^{17}$, Roberto Furlanetto Júnior ${ }^{18}$, Camilo Luis Monteiro Lourenço ${ }^{19}$ and Giuseppe Biondi-Zoccai ${ }^{20}$

${ }^{1}$ Federal University of Uberlândia, Department of Clinical Research, Heart Institute (InCor), Master Institute of Education President Antonio Carlos, IMEPAC, Araguari, Brazil

${ }^{2}$ HCFMUSP- University of São Paulo Medical School, Department of Cardiology, São Paulo, Brazil

${ }^{3}$ Faculty of Medicine ABC, Department of Cardiology Santo André, Brazil

${ }^{4}$ Cardiovascular Research Center, ShahidSadoughi University of Medical Sciences, Department of Cardiology, Yazd, Iran

${ }^{5}$ Department of Specialized and General Surgery, Fluminense Federal University, Rio de Janeiro, Brazil

${ }^{6}$ Dante Pazzanese Institute of Cardiology,Department of Clinical Research, São Paulo, Brazil

${ }^{7}$ Graduate Program in Medicine and Health, Department of Health and Sciences, Federal University of Bahia, Brazil

${ }^{8}$ Federal University of Minas Gerais, Department of Cardiology, MG, Brazil

${ }^{9}$ Federal University of Mato Grosso, Department of Medicine, MT, Brazil

${ }^{10}$ FOP Unicamp, Department of Clinical Research, SP, Brazil

${ }^{11}$ Division of Cardiology, Duke University Medical Center, Department of Clinical Research, Durham, NC, USA

${ }^{12}$ Monash Cardiovascular Research Centre and Monash Heart, Department of Cardiology, Clayton, Victoria, Australia

${ }^{13}$ Tehran University of Medical Sciences, Department of Medicine, Iran

${ }^{14}$ University of Connecticut/Hartford Hospital Evidence-Based Practice Center, Hartford, Department of Comparative Effectiveness and Outcomes Research Health Outcomes, CT, USA

${ }^{15}$ Laboratory of Epidemiology, University of Extremo Sul Catarinense, Criciúma, Brazil

${ }^{16}$ Department of Medicine and Therapeutics and Li Ka Shing Institute of Health Sciences, Faculty of Medicine, Chinese University of Hong Kong, Hong Kong, China

${ }^{17}$ Tianjin Key Laboratory of Ionic-Molecular Function of Cardiovascular Disease, Department of Cardiology, Tianjin Institute of Cardiology, the Second Hos-pital of Tianjin Medical University, Tianjin, China

${ }^{18}$ Department of Medico-Surgical Sciences and Biotechnologies, Sapienza University of Rome, Latina \& Department of AngioCardioNeurology, IRCCS Neuromed, Pozzilli, Italy ${ }^{19}$ Department of Medico-Surgical Sciences and Biotechnologies, Sapienza University of Rome, Latina, Italy

${ }^{20}$ Department of AngioCardioNeurology, IRCCS Neuromed, Pozzilli, Italy

\begin{abstract}
Introduction: Epicardial adipose tissue (EAT) is a complex disease that includes endothelial dysfunction, insulin resistance, diabetes, hypertension, ectopic obesity, and dyslipidaemia and an increased risk of cardiovascular events. This study aims to fill this gap of research by conducting a systematic review and meta-analysis to compare differences types of exercise to reduce EAT.

Methods and analysis: We will search the PubMed, EMBASE, Cochrane Library, Web of Science, Embase, google scholar, clinical trials registry (ClinicalTrials. gov) for unpublished or undergoing research listed in registry platforms. Randomized controlled trials (RCTs) on the drug therapy of EAT with outcome measures including diagnostic criteria of EAT will be included. The quality of included RTCs will be evaluated according to the Cochrane Collaboration's risk of bias tool. Sensitivity analysis on the sample size of RCTs, meta-regression analysis on the follow-up periods, dosages and baselines of outcome measure, contradiction analysis between pairwise and network meta-analyses, and publication bias analysis, will be performed. The quality of included RTCs will be evaluated according to the Cochrane Collaboration's risk of bias tool. Sensitivity analysis on the sample size of RCTs, meta-regression analysis on the follow-up periods, dosages and baselines of outcome measure, contradiction analysis between pairwise and network meta-analyses, and publication bias analysis, will be performed.
\end{abstract}

Ethics and dissemination: Ethics approval was not required for this study because it was based on published studies and this study will be submitted in a scientific peer-reviewed journal.

Trial registration number: PROSPERO (CRD42018096581).

${ }^{*}$ Correspondence to: Leonardo Roever, Department of Clinical Research, Federal University of Uberlândia, Heart Institute (InCor), Master Institute of Education President Antonio Carlos, IMEPAC, Araguari, Brazil, E-mail: leonardoroever@hotmail.com

Key words: exercise, epicardial fat, systematic review

Received: March 15, 2019; Accepted: March 26, 2019; Published: March 29, 2019 
Abbreviations: Cis: Confidence Intervals; HDL: High Density Lipoprotein; LDL: Low Density Lipoprotein; MD: Mean Difference; RR: Risk Ratio, WC: Waist Circumference; EAT: Epicardial Adipose Tissue; MetS: Metabolic Syndrome; CVD: Cardiovascular Disease; NMA: Network Meta-analysis, RCTs: Randomized Controlled Trials.

\section{Strengths and limitations of this study}

This systematic review and meta-analysis will offer better understanding regarding the association between exercise and EAT. Included studies may have substantially different methodologies, which could limit our ability to draw reliable conclusions from the existing evidence base. Depending on the results, confounding factors that were not adjusted for in the selected studies and low generalizability can be limitations. Individual patient data will not be available.

\section{Background}

Epicardial adipose tissue (EAT) is associated with endothelial dysfunction, insulin resistance, diabetes, hypertension, ectopic obesity, and dyslipidemia and an increased risk of cardiovascular disease and stroke [1-4]. It is in large part the result of unbalanced diet, low socioeconomic and cultural level, stress and sedentary lifestyle [3-8].

Currently, there is a lack of high-quality evidence to support the tailoring of drugs to treatment regimens for EAT according to an individual's characteristics or other treatment effect modifiers. As such, it is imperative to improve existing guideline recommendations and inform decision-makers about the safety and effectiveness of these interventions. This study conducted a Systematic review and metaanalyses compare the differences types of exercise to reduce EAT.

\section{Objective}

The objective of this study is to compare efficacies of exercise to reduce EAT.

\section{Methods/design}

Systematic review and meta-analyses of EAT deposit of visceral fat, located between the heart and the pericardium, which shares many of the pathophysiological properties of other visceral fat deposits, but with potential direct local effects in the inflammatory and coronary atherosclerotic process $[9,11]$.

The study is registered with PROSPERO (CRD42018096581). This protocol conforms to the Preferred Reporting Items for Systematic Reviews and Meta-Analyses Protocols (PRISMA-P) guidelines [12].

\section{Eligibility criteria}

We will include randomized controlled trials (RCTs) comparing one drug with another drug as monotherapy or placebo for the treatment of TN. Quasi-randomized controlled trails allocating participants according to birth date or the consequences of enrolment will be excluded. The minimum duration for RCT inclusion will be set at 4 weeks. Trials with more than a two-arm design will be considered only if the available data meet the criteria for an intervention. For trials with a crossover design, data will only be extracted from the first randomization period. The retrieved reports will be screened according to the checklist of eligibility and the eligibility criteria shown below including participants, interventions, controls, types of study and other criteria.

Patient and Public Involvement: The patients and/or the public are not involved; it is an article of protocol of systematic revision and network of meta-analysis.

\section{Search strategy}

The following databases will be searched from their inception forward for potentially eligible studies in English language published on or before 31 January 2018: (1) PubMed, (2) Scopus, (3) Web of Science, (4) Cochrane Central Register of Controlled Clinical Trials, (5) Embase, (6) google scholar, (7) clinical trials registry (ClinicalTrials. gov). In addition, cross-referencing from retrieved studies will be conducted. As publication bias caused by insufficient unpublished data can significantly bias the comparative efficacy results of network meta-analyses and modify rankings, we will also perform searches for unpublished or ongoing trials using the System for information on Grey Literature in Europe (SIGLE) as well as other registry platforms, such as Clinicaltrials.gov and the International Clinical Trials Registry Platform. Prior to completing this review, we will perform an additional search of each database and registration platform to guarantee that the most recent studies are included. Electronic databases will be searched for studies on the effects of drugs on risk factors safety in adults with MetS. The first author will conduct all database searches. The search strategy for all other databases will be adapted based on the requirements of each database. The search strategy will be developed by LR and HRZ; we anticipate that the databases will be searched from their inception to 30 December 2018, the search strategies for PubMed, EMBASE, Cochrane Library, Web of Science, Embase, google scholar, clinical trials registry (ClinicalTrials.gov).

\section{Participants}

\section{Inclusion}

The participants must be adults, aged at least 18 years, suffering from and requiring treatment pharmacology for EAT. Measurement of epicardial adipose tissue (EAT) by linear or volumetric analysis using MRI, CT or echocardiography. Patients who have undergone a full 3D assessment of the myocardium in order to obtain full volumetric measurement of the epicardial adipose tissue. This can only be performed with MRI or CT imaging. Echocardiography as an imaging modality to obtain myocardial function, specifically measures of systolic function (e.g. ejection fraction), diastolic function (e', E/A ratio and other Doppler derived indices, as well as global longitudinal strain analysis), or full volume cardiac CT or MRI imaging to obtain ventricular volumes function parameters.

\section{Exclusion}

The participants suffering from other metabolic disease conditions or aged less than 18 years. Studies without an exercise or physical activity component. Narrative reviews, systematic reviews, opinion papers, letters and any publication without primary data and/ or explicit description of the methods. Duplicate publications from the same study. For those studies published in more than one journal/ conference, the most comprehensive and recent report will be used.

\section{Interventions}

\section{Other criteria}

Other inclusion criteria: The RCTs must report complete efficacy data of risk factors of each treatment. Follow-up periods or durations in RCTs are at least 4 weeks. Other exclusion criteria are (1) duplicated or redundant studies and (2) combined treatments with multiple drugs. 


\section{Study selection}

Reviewers will screen all titles or abstracts or full texts for database records independently according to the eligibility criteria. Disagreements between reviewers will be resolved by consensus. Selection process of relevant studies retrieved from databases will be shown in a PRISMA-compliant flow chart (Figure 1) [12].

\section{Comparator(s)/control}

The change in the thickness of the epicardial fat after the exercise program.

\section{Context}

\section{Main outcome(s)}

The change in the thickness of the epicardial fat. Secondary outcomes will include body weight, lean body mass, waist circumference, waist-to-hip ratio, energy intake, energy expenditure, physical activity level, maximum oxygen consumption (relative and absolute), muscular strength, resting systolic and diastolic blood pressures, total cholesterol, high-density lipoprotein cholesterol, ratio of total cholesterol to high-density lipoprotein cholesterol, non-highdensity lipoprotein cholesterol, low-density lipoprotein cholesterol,

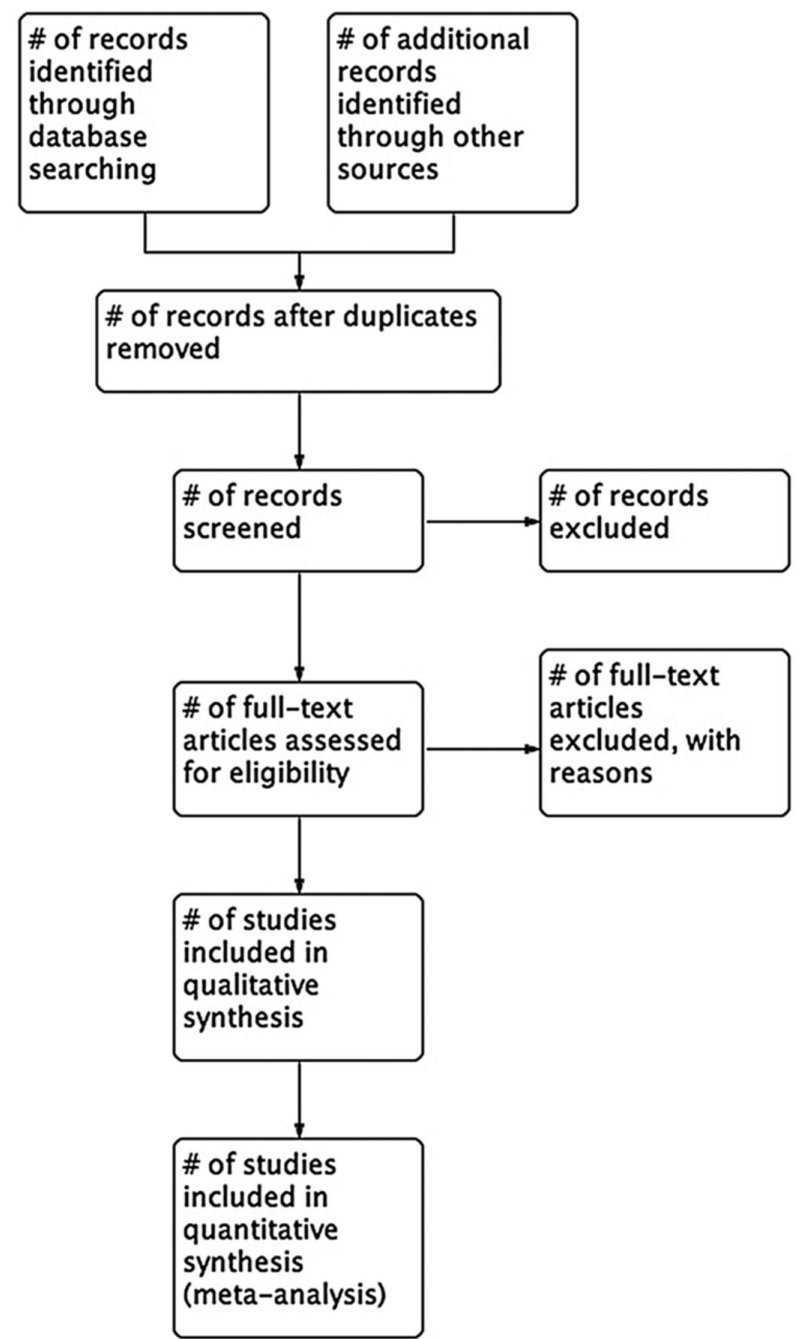

Figure 1. Flow diagram of study selection process triglycerides, glycosylated haemoglobin, fasting and non-fasting glucose and insulin.

\section{Timing and effect measures}

Pre-to-post intervention changes.

\section{Additional outcome(s)}

The change in risk factors for atherosclerosis.

\section{Timing and effect measures}

Pre-to-post intervention changes.

\section{Data extraction (selection and coding)}

Two independent investigators will review study titles and abstracts, and studies that in accordance with the inclusion criteria will be retrieved for full-text assessment. Trials selected for detailed analysis and data extraction will be analyzed by two investigators. Disagreements will be resolved by a third investigator.

\section{Risk of bias (quality) assessment}

The methods set out in the Cochrane Handbook for Systematic Reviews of Interventions will be used to assess the risk of bias of the included studies. This assessment will be carried out independently by two members of the research team. Disagreements will be resolved by a third investigator. We will use a score based on the Cochrane Collaboration tool for assessing risk of bias for each included study and we will use the GRADE approach to assess the quality of the evidence produced by this systematic review.

\section{Strategy for data synthesis}

The data of interest presented as continuous (mean value and $\mathrm{SD})$ will be used to perform meta-analysis to obtain the standardized mean difference (SMD) and 95\% confidence interval (CI). Cochran's Q-statistic and $\mathrm{I}^{2}$ test will be used to test for heterogeneity between the included studies. If $\mathrm{I}^{2}$ value is greater than $50 \%$ or a $\mathrm{p}$ value of the Q-test is less than 0.05 , indicating maximal heterogeneity among the included studies, a random-effects model will be used. Aggregate data extracted from included studies will be used for quantitative synthesis.

\section{Analysis of subgroups or subsets}

The subgroup meta-analyses will be conducted according to the pre-specified study-level characteristics using a fixed-effect meta-analysis and if there is substantial heterogeneity, we will use the random effects model. The sources included location, sex, age, method of EAT assessment, the type of exercise. We will also conduct sensitivity analyses to evaluate the potential sources of heterogeneity in the analyses. Potential sub-analysis will include baseline participant characteristics (e.g. age, sex and initial body mass index) and exercise (modality) intervention characteristics.

\section{Amendments}

If it is necessary, we will update this protocol in the future. We will submit the original protocol, final protocol and summary of changes as a supplement.

\section{Ethics and dissemination}

\section{Ethical issues}

No ethical approval is required because this study includes no confidential personal data or interventions with the patients. 


\section{Publication plan}

The procedures of this systematic review and NAM will be conducted in accordance with the PRISMA-compliant guideline. The results of this systematic review and NAM will be submitted to a peerreviewed journal for publication.

\section{Authors' information}

Not applicable.

\section{Competing interests}

The authors declare that they have no competing interests.

\section{Consent for publication}

Not applicable.

\section{Ethics approval and consent to participate}

Not applicable.

\section{Availability of supporting data}

Not applicable.

\section{Funding}

Not applicable.

\section{Authors' contributions}

LR, ASRB, ALDD, ACF, NPS, PMMD, RMLS, JLO, MN, AD, PARD, FRS, GBFO, GT, GBZ, SAH, PEOR, AJG, RMP, ACF, CLML, PMMD, TMF, NN, SA, CD, PFSG, A.A, AVH, MIR, RDL, RFJ and FCV conceived the study idea and devised the study methodology. LR, ASRB, ACPC and ESR participated in the design and coordination of the study. LR was primarily responsible for protocol writing and developed the search strategy. LR and FCV will screen identified literature, conduct data extraction and analyses the review findings. All authors read the drafts, provided comments and agreed on the final version of the manuscript.

\section{Acknowledgements}

Not applicable.

\section{References}

1. Basurto Acevedo L, Barrera Hernández S, Fernández Muñoz MJ, Saucedo García RP, Rodríguez Luna AK, et al. (2018) An increase in epicardial fat in women is associated with thrombotic risk. Clin Investig Arterioscler 30: 112-117. [Crossref]

2. Oliveira GB, Avezum A, Roever L (2015) Cardiovascular disease burden: evolving knowledge of risk factors in myocardial infarction and stroke through population-based research and perspectives in global prevention. Front Cardiovasc Med 2: 32. [Crossref]

3. Roever L, Biondi-Zoccai G2, Chagas AC3 (2016) Non-HDL-C vs. LDL-C in Predicting the Severity of Coronary Atherosclerosis. Heart Lung Circ 25: 953-954. [Crossref]

4. Roever LS, Resende ES, Diniz ALD, Penha-Silva N, Veloso FC, et al. (2016) Abdominal obesity and association with atherosclerosis risk factors. Medicine (Baltimore) 95: e1357. [Crossref]

5. Roever L, Resende ES, Diniz AL, Penha-Silva N, Veloso FC, et al. (2015) Ectopic adiposopathy and association with cardiovascular disease risk factors: The Uberlândia Heart Study. Int J Cardiol 190: 140-142. [Crossref]

6. Roever L, Resende ES, Veloso FC, Diniz AL, Penha-Silva N, et al. (2015) Perirenal Fat and Association With Metabolic Risk Factors: The Uberlândia Heart Study. Medicine (Baltimore) 94: e1105. [Crossref]

7. van Rooy MJ, Pretorius E (2015) Metabolic syndrome, platelet activation and the development of transient ischemic attack or thromboembolic stroke. Thromb Res 135: 434-442. [Crossref]

8. Sarrafzadegan N, Gharipour M, Sadeghi M, Nezafati P, Talaie M, et al. (2017) Metabolic Syndrome and the Risk of Ischemic Stroke. J Stroke Cerebrovasc Dis 26: 286-294. [Crossref]

9. Sánchez-Iñigo L, Navarro-González D, Fernández-Montero A, Pastrana-Delgado J, Martínez JA (2017) Risk of incident ischemic stroke according to the metabolic health and obesity states in the Vascular-Metabolic CUN cohort. Int J Stroke 12: 187-191. [Crossref]

10. Chei CL, Yamagishi K, Tanigawa T, Kitamura A, Imano H, et al. (2008) Metabolic Syndrome and the Risk of Ischemic Heart Disease and Stroke among Middle-Aged Japanese. Hypertens Res 31: 1887-1889. [Crossref]

11. Fang X, Liu H, Zhang X, Zhang H, Qin X, et al. (2016) Metabolic Syndrome, Its Components, and Diabetes on 5-Year Risk of Recurrent Stroke among Mild-toModerate Ischemic Stroke Survivors: A Multiclinic Registry Study. Stroke Cerebrovasc Dis 25: 626-634. [Crossref]

12. Glasziou P, Irwig L, Bain C, Colditz G (2001) Systematic reviews in health care: practical guide. Cambridge: Cambridge University Press.

Copyright: (C2019 Roever L. This is an open-access article distributed under the terms of the Creative Commons Attribution License, which permits unrestricted use, distribution, and reproduction in any medium, provided the original author and source are credited. 